\title{
ARRIVAL TIME VALUATION OF COMMUTERS IN URBAN RAIL TRANSIT
}

\author{
Yan $\mathrm{CHENG}^{1^{*}}$, Xiafei $\mathrm{YE}^{2}$, Zhi WANG ${ }^{3}$ \\ ${ }^{1}$ Centre for Transport Studies, University College London, London, United Kingdom \\ ${ }^{2,3}$ MOE Key Laboratory of Road and Traffic Engineering, Tongji University, Shanghai, China
}

Received 26 December 2017; revised 23 April 2018, 29 May 2018; accepted 17 July 2018

\begin{abstract}
Departure time choice of commuters is one of key decisions affecting the crowding of urban rail transit network during peak hours. It is influenced by arrival time value, the additional psychological pressure caused by in-vehicle crowding, and time uncertainty. This paper aims at investigating how commuters in urban rail transit value their arrival time at work/school. Three valuation frameworks are proposed based on the reference point approach of prospect theory. Non-linear value functions with different reference point alternatives are estimated using data from a survey and stated choice study of users of Shanghai Metro system. Results show that schedule delay with work/school start time as the only reference point cannot properly reflect the arrival time valuation of urban rail transit commuters. Instead, the valuation framework with preferred arrival time as a reference point fits best, which hits as much as $85.64 \%$ of the cases. The asymmetrical response to early-side and late-side arrivals is identified. The findings of this study provide an essential basis for the development of departure time choice model.
\end{abstract}

Keywords: urban rail transit, commuter, departure time choice, arrival time value, reference point, valuation framework.

\section{Introduction}

Urban rail transit is playing a more important role in urban transportation of many cities because of its speediness, punctuality and large capacity. It undertakes a large number of medium- and long- distance commutes during morning and evening peak hours. As a result, the quality of rail transit is becoming a severe problem. In several metropolitan areas, trains are overcrowding and it is hard for passengers to get onto the metro trains at peak times. Both route choice and departure time choice of commuters are key decisions affecting the crowding of urban rail transit network. However, commuter departure time choice is usually ignored or assumed to be given in transit assignment models (Poon et al. 2004; Wu, Liu 2004; Huang 2010; Liu 2012, 2013; Yang 2013).

A considerable amount of research effort has been devoted to departure time choice in road traffic since the 1980s, both in econometric modeling (Abkowitz 1980; Small 1982; Hendrickson, Plank 1984; Noland, Small 1995; Kristoffersson 2007; Jou et al. 2008; Van de Kaa 2010; Yang, Liu 2018), and dynamic user equilibrium analysis fields (De Palma et al. 1983; Mahmassani, Chang 1986; Arnott et al. 1990; Ran et al. 1996; Yang, Meng 1998; Huang, Lam 2002; Han et al. 2011). The key problem is to explore the relation between arrival time value, which is usually interpreted as schedule delay, and the time uncertainty caused by congestion. However, in urban rail transit, the increase of passengers has no effect on the speed, so in-vehicle time remains unchanged. Instead, the growing number of passengers leads to the aggravation of in-vehicle crowding, the extension of boarding and alighting time, and the extra waiting time for the passengers who cannot board the first incoming train. Thus, what affects departure time choice in urban rail transit is different from the ones in road traffic. The influence factors are mainly composed of arrival time value, the additional psychological pressure caused by in-vehicle crowding, and time uncertainty.

In the past two decades, departure time choice in urban rail transit has received more attention (Ieda et al. 2002; Soyama et al. 2010; Feng et al. 2013; Peer et al. 2016; Yang, Tang 2018). Most of researches agree with the viewpoint that departure time choice of urban rail commuters is a trade-off between schedule delays and crowding (Harada et al. 2002; Tian, Huang 2004; Iwakura, Harada 2005; Tian et al. 2007; Wu, Huang 2009; De Palma et al. 2015, 2017). Although crowding is very important, this

${ }^{*}$ Corresponding author. E-mail: yan.cheng@ucl.ac.uk 
paper focuses on the arrival time value of commuters in urban rail transit. The arrival time of a commuter is a specific moment in one day, but its value mainly depends on its deviation from the commuter's reference time. Due to distinctive traveller characteristics, it seems almost impossible to assume that commuter drivers and commuter rail transit users have a same understanding of time. So the functional form of arrival time value is needed to be investigated according to the responses of commuters in urban rail transit.

In most of existing studies, the arrival time value of commuters is converted into schedule delay. The concept of schedule delay was proposed by Vickrey (1969) to measure the disutility of arriving at the destination earlier or later than preferred. When a commuter arrives at his/ her work/school place at desired arrival time, the schedule delay equals to zero; otherwise, the commuter has a schedule delay early or schedule delay late. The desired arrival time is assumed to be official Work/school Start Time (WST) in most of previous researches (Harada et al. 2002; Ieda et al. 2002; Iwakura et al. 2003; Tian, Huang 2004; Iwakura, Harada 2005; Tian et al. 2007; Wu, Huang 2009; Yang, Liu 2018; Yang, Tang 2018). Recently, researchers try to relax this assumption (De Palma et al. 2015, 2017; Peer et al. 2016). However, there are very few studies about how this time should be fixed. On the other hand, the most common expression of schedule delay used in quantitative analysis is linear function (Harada et al. 2002; Iwakura et al. 2003; Tian, Huang 2004; Iwakura, Harada 2005; Tian et al. 2007; Wu, Huang 2009), which means commuters show the same response to every unit schedule delay with the increase of the difference between Actual Arrival Time (AAT) and desired arrival time. Only a few scholars noticed that commuters may change their risk attitude to the loss. Ieda et al. (2002) use a non-linear function to depict schedule delay late, while Soyama et al. (2010) use a quadratic function. Both functions are convex, so schedule delay grows faster when commuters begin to suffer late arrival, and gradually become stable. However, these functions are predetermined without estimation of exponent, so the risk attitude of commuters in urban rail transit when they are facing different extents of loss remains an open research question.

According to qualitative analysis, both schedule delay early and late should have negative effects in departure time choice, which is proved by many works (Harada et al. 2002; Iwakura et al. 2003; Iwakura, Harada 2005; Peer et al. 2016). However, the relationship between two parameters themselves is not clear. Iwakura et al. (2003) establishes departure time choice models based on Multinomial Logit Model (MNL) and Mixed Logit Model (MXL). Both models are estimated with the SP and RP data of commuters in Tokyo rail transit system, and results show that the disutility of unit schedule delay late is greater than the one of schedule delay early. However, Peer et al. (2016) provides a conflicting result. The proposed MNL model is estimated with the data of regular train users in the Netherlands, and the value of schedule delay early during the morning commute is higher than the one of schedule delay late. The different choice of desired arrival time in two studies may be one of the reasons for this contradiction. However, it also reminds us that the attitudes of commuters toward early arrival and late arrival need more focus.

So many researches use schedule delay as a proxy of the arrival time value of commuter, leading to the misunderstanding of arrival time value. When a commuter judges his/her arrival time, several criteria may be used. Therefore, arrival time value may consists of different parts. Soyama et al. (2010) first splits arrival time value into two parts, the deviation penalty and late arrival penalty. The former one takes Preferred Arrival Time (PAT) as its criterion, while the latter one's criterion is WST.

Although these studies provide an insight into the valuing method of commuters' arrival time in urban rail transit, there are two drawbacks. First, the choice of criteria that commuters using in valuing arrival time is so limited, only WST and desired arrival time. Second, the influence of psychological factors (such as risk attitude) on the valuation of commuter arrival time has not been discussed.

To overcome these shortcomings, the knowledge about decision-making under uncertainty is indispensable. Prospect theory proposed by Kahneman and Tversky (1979) is usually used to analyse this issue. It is based on the assumption that people are "bounded rational" (Simon 1955) instead of "complete rational", owning to their limited cognitive ability, inferential capability and information acquisition. The value function of prospect theory is used to evaluate each possible result, reflecting the subjective values of different results. The function is defined on the deviation from reference points, which means it focuses on the magnitude of change from reference points. This determining method is called reference point approach. The value function has already been introduced to commuter departure time choice model in road traffic. A foursegmented value function is developed by Jou and Kitamura (2002). The reference points of this value function are the earliest arrival time, the PAT, and the WST for a given commuter. Using the maximum likelihood method, Jou et al. (2008) estimate the value model with the survey data of auto commuter departure time decision. Limited to the tool, the value function is simplified to a linear form. Results indicate that the commuter behaviour is consistent with the theoretical postulates of prospect theory. However, it cannot examine the concavity and convexity of the function. Two valuation frames that comply with the prospect theoretic propositions are compared by Senbil and Kitamura (2004). Empirical results suggest that prospect theory is applicable in the departure time choice of commuter drivers. However, whether it is applicable to commuter rail transit users remains unknown.

The goal of this paper is to examine the applicability of the reference point hypothesis of prospect theory to ar- 
rival time valuation of urban rail commuters. We aim to address the following questions:

- what reference points are chosen by commuters to measure their arrival time value?

- does schedule delay with WST as the only reference point represent the arrival time value of commuters in urban rail transit properly?

- do commuters judge their arrival time value in a linear way?

- if not, how do risk attitude influence their valuation?

- which situation do commuters attach more disutility to?

- being early or being late?

This paper gives a clear solution to these problems. The reference point approach of prospect theory is introduced. In addition, three valuation frameworks with various reference points are proposed. For each kind of reference point, there are several alternatives. Different combinations of valuation framework and reference point alternatives will be empirically tested and compared with the data of commuters in Shanghai Metro System. The risk attitude of urban rail commuters is also revealed by estimation results.

The remainder of the study is organized as follows. Section 1 introduces some background on the reference point approach. Three valuation frameworks and several alternative reference points are proposed. Section 2 presents survey design and the data of Shanghai Metro commuters. The relationships among reference point alternatives are analysed. Section 3 estimates the models within each framework and discusses the results. Finally, main conclusions are summarized in the last section.

\section{Methodology}

According to prospect theory, a commuter is assumed to maintain the same choice as long as his/her AAT is within an indifference band; otherwise, the commuter will adjust his/her departure time through some procedures. This conclusion is confirmed by Chang and Mahmassani (1989) in road traffic. In prospect theory, the value function is used to reflect the subjective value of each possible result. It is a function of the deviation from reference points, so the focus is the magnitude of change from reference points, instead of the absolute value. This determining method is called reference point approach. Based on prospect theory, the value function has other two properties. First, it is generally concave for gains and convex for losses. Second, it is steeper for losses than for gains. These two properties is consistent with the standpoints of prospect theory that commuters react asymmetrically to gains and losses, and they exhibit risk aversion for gains and risk seeking for losses.

The reference point approach in the value function of prospect theory is introduced in this paper to valuing the arrival time of commuters in urban rail transit. The assumption that commuters maintain their departure time when their AAT is within an indifference band is also acceptable. However, the concavity and convexity of value function is not predetermined. A value function is defined by a valuation framework and the value of reference points. Three valuation frameworks with various reference points as well as several reference point alternatives are proposed. Whether these arrival time value functions of commuter rail transit users are in accordance with prospect theory needs to be verified by empirical studies.

\subsection{Valuation frameworks}

Valuation framework is a useful tool in elucidating the behaviour patterns of commuters in urban rail transit. Based on reference point approach, the value of a commuter's arrival time is defined as a function of the difference between their AAT and reference points. Therefore, reference point plays a crucial role in the determination of a valuation framework. The prospect theory assumes that a commuter maintains the same choice as long as his/ her AAT is within an indifference band. The demarcation points of the indifference band are defined as zero-value positions. When a commuter arrives at these moments, arrival time value equals to zero. The lower limit position is called the demarcation point of too-Early Arrival Time (EAT), and the upper limit position is called the demarcation point of too-Late Arrival Time (LAT). Furthermore, a commuter experiences the maximum value when he/ she arrives at work place/ school at extreme-value position. This moment is defined as Optimal Arrival Time (OAT) at work place/ school. One or both kinds of these reference points are involved in our following valuation frameworks.

An arrival is defined as an early-side arrival if AAT is earlier than OAT, and it is defined as a late-side arrival if AAT is later than OAT. Three valuation frameworks are proposed in this paper according to diverse behaviour assumptions of commuters who are early-side arrivals and late-side arrivals, which are described as follows:

- F1: Commuters value their arrival time according to the demarcation points of indifference band;

- F2: Commuters value their arrival time only according to OAT;

- F3: Commuters value their arrival time according to OAT when they are early-side arrivals, and value their arrival time according to the demarcation point of LAT when they are late-side arrivals.

Framework F1 is developed by Jou and Kitamura (2002). EAT and LAT are reference points, and OAT is a "pseudo" reference point to define early-side and lateside arrival. The conceptual diagram illustrated in Figure 1 shows a gain occurs when a commuter's arrival time is within the range of two reference points (segments II and III), and a loss occurs when the commuter experiences an arrival time which is beyond this range (segments I and IV). The reference point for an early-side arrival is EAT, and for a late-side arrival is LAT. 
Within framework F2, only OAT is used to value arrival time. Arrival time value reaches the maximum at the reference point, and decreases with the difference between AAT and the reference point, both on early-side (segments I) and late-side (segments II), as shown in Figure 2.

Framework F3 is a combination of F1 and F2. The situation of an early-side arrival is similar to F2. When a commuter arrival time is earlier than OAT, a gain or a loss may occur according to the deviation relative to OAT. Meanwhile, the situation of a late-side arrival is similar to F1. A gain occurs when a commuter's arrival is between OAT and LAT. Moreover, a loss occurs when arrival time is later than LAT (Figure 3).

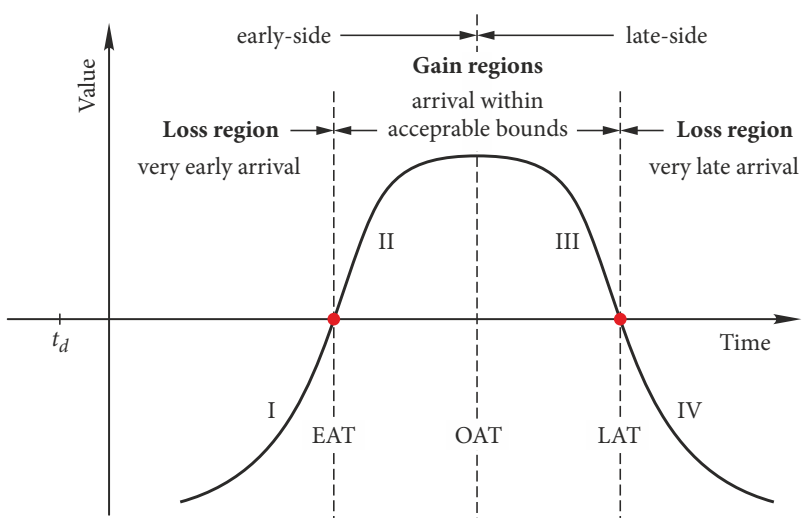

Figure 1. Diagrammatic sketch of arrival time value framework 1

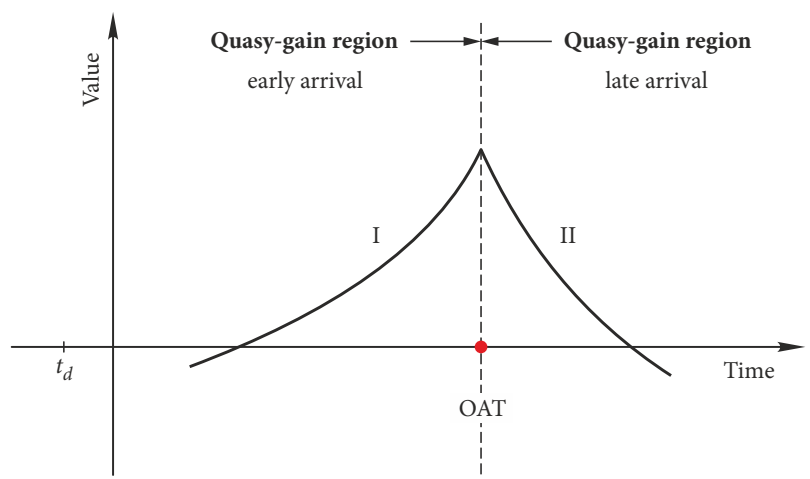

Figure 2. Diagrammatic sketch of arrival time value framework 2

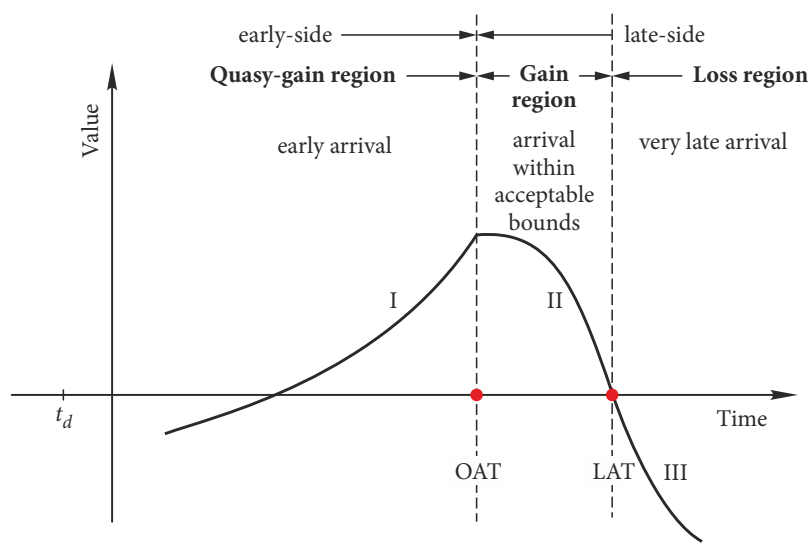

Figure 3. Diagrammatic sketch of arrival time value framework 3
Value function is assumed to be a random function due to the cognitive limitation of commuters. The segmented value functions for a commuter according to F1 to F3 are given in Equations (1)-(3):

$$
\begin{aligned}
& V_{1}\left(t_{a}\right)= \begin{cases}\beta_{1} \cdot\left(t_{E}-t_{a}\right)^{\alpha_{1}}+\varepsilon_{e}^{L}, & t_{d}<t_{a} \leq t_{E} \\
\beta_{2} \cdot\left(t_{a}-t_{E}\right)^{\alpha_{2}}+\varepsilon_{e}^{G}, & t_{E}<t_{a} \leq t_{O} \\
\beta_{3} \cdot\left(t_{L}-t_{a}\right)^{\alpha_{3}}+\varepsilon_{l}^{G}, & t_{O}<t_{a} \leq t_{L} \\
\beta_{4} \cdot\left(t_{a}-t_{L}\right)^{\alpha_{4}}+\varepsilon_{l}^{L}, & t_{a} \geq t_{L}\end{cases} \\
& V_{2}\left(t_{a}\right)= \begin{cases}\gamma_{1}+\beta_{5} \cdot\left(t_{O}-t_{a}\right)^{\alpha_{5}}+\varepsilon_{e}, & t_{d}<t_{a} \leq t_{O} ; \\
\gamma_{1}+\beta_{6} \cdot\left(t_{a}-t_{O}\right)^{\alpha_{6}}+\varepsilon_{l}, & t_{a}>t_{O} ;\end{cases} \\
& V_{3}\left(t_{a}\right)= \begin{cases}\gamma_{2}+\beta_{7} \cdot\left(t_{O}-t_{a}\right)^{\alpha_{7}}+\varepsilon_{e}, & t_{d}<t_{a} \leq t_{O} ; \\
\beta_{8} \cdot\left(t_{L}-t_{a}\right)^{\alpha_{8}}+\varepsilon_{l}^{G}, & t_{O}<t_{a}<t_{L} ; \\
\beta_{9} \cdot\left(t_{a}-t_{L}\right)^{\alpha_{9}}+\varepsilon_{l}^{L}, & t_{a} \geq t_{L},\end{cases}
\end{aligned}
$$

where: $t_{d}$ is departure time; $t_{a}$ is AAT; $t_{O}$ is OAT; $t_{E}$ is the demarcation time of EAT; $t_{L}$ is the demarcation time of LAT.

The error terms are assumed to be normally distributed with zero means and heteroskedastic variances under various circumstances. They are distinguished by subscripts and superscripts. The subscripts $e$ and $l$ represent early-side and late-side, respectively, and the superscripts $G$ and $L$ represent gain $(V>0)$ and loss $(V \leq 0)$, respectively. Parameters $\alpha_{i}(i=1, \ldots, 9)$ represent the rate of changes in value to the commuter. The signs and values of $\alpha_{i}(i=1, \ldots, 9)$ are not predetermined. Parameters $\beta_{i}(i=1, \ldots, 9)$ are weights, which represent the importance of value in different segments to the commuter. According to the frameworks, except $\beta_{2}, \beta_{3}$ and $\beta_{8}$, the other $\beta$ should take on negative values. Parameters $\gamma_{i}(i=1,2)$ are the values of OAT within framework F2 and F3, which should be positive. The parameters $\alpha, \beta$ and $\gamma$ are to be estimated.

\subsection{Alternative reference points}

Two demarcation points of indifference band (EAT and LAT) and OAT are taken as reference points within three frameworks. However, their values are not determined. In this paper, each reference point has several alternatives.

The Acceptable Earliest Arrival Time (AEAT) and WST are the alternative to EAT and LAT in road traffic departure time choice studies (Jou, Kitamura 2002; Senbil, Kitamura 2004; Jou et al. 2008). Both of them are reserved. In view of that, more commuters are allowed to be later than WST, so the Acceptable Latest Arrival Time (ALAT) is introduced as an alternative to LAT in this paper. Furthermore, based on the concept of indifference band, we first propose the time when a commuter Starts to Consider Departing Later Time (SCDLT) and the time, when a commuter Starts to Consider Departing Earlier Time (SCDET) as alternatives to EAT and LAT, respectively. It needs to be verified by survey data whether these 
two alternatives have similar values to other alternatives of EAT and LAT.

For OAT, the first alternative is the PAT at work place/ school of commuters. To examine whether the schedule delay with WST as the only reference point is the equivalent of arrival time value, WST is taken as the other alternative to OAT within framework F2.

In summary, EAT has two alternatives (AEAT and SCDLT), while LAT has three alternatives (WST, ALAT and SCDET). The value of OAT within F1 and F3 is PAT. When the value function is based on framework F2, then both PAT and WST are the alternatives to OAT. Different combinations of valuation framework and reference point alternatives will be tested and compared with empirical data.

\section{Data}

\subsection{Survey}

The dataset used for the estimation of arrival time value function was collected in 2017. An online questionnaire was randomly mailed to around 1400 people who are commuter rail transit users in Shanghai (China). A questionnaire was regarded as valid only if a respondent went to work/school by urban rail transit in the first trip on the latest weekday. 585 questionnaires left after a validity check.

Except socioeconomic properties and travel information, the survey asked commuters about the key points of their arrival time valuation, including WST, AEAT, ALAT, PAT, SCDLT and SCDET. Moreover, in the stated choice experiment, respondents were inquired for their willingness to switch departure time according to their AAT on the latest weekday.

The socioeconomic and travel characteristics of the sample are presented in Table 1. Gender is evenly distributed in the valid samples. Females accounts for 54.53\%, while males accounts for $45.47 \%$. A large majority of respondents are aged between 18 and 40 years old (87.69\%).
$89.57 \%$ of respondents travel by metro more than 3 times in one week, which suggests that the respondents are familiar with Shanghai Metro network. For most of respondents (97.09\%), going to work is their purposes of the first metro trip on the latest weekday. The WST of most of respondents is within 7:30...9:30 am (96.24\%). A majority of respondents start work/school at 9:00 am (55.55\%), followed by $8: 30$ am (22.13\%), as shown in Figure 4 .

\subsection{Reference points analysis}

The statistics of alternative reference points for urban rail transit commuters surveyed are shown in Table 2. The ANalysis Of VAriance (ANOVA) result in Table 3 shows that except the difference between WST and SCDET, all the others between alternative reference points are significant at the 5\% significance level. For the demarcation point of EAT, the average of SCDLT is 5.76 min earlier than the average of AEAT. $89.57 \%$ of respondents start to consider departing later when time is not later than AEAT. On the other hand, for the demarcation point of LAT, the average of WST is the earliest one among three alternatives, which is 8:48 am. 59.49\% of respondents are allowed to be late for work, and the average of ALAT is $7.27 \mathrm{~min}$ later than the average of WST. So tardiness should not be

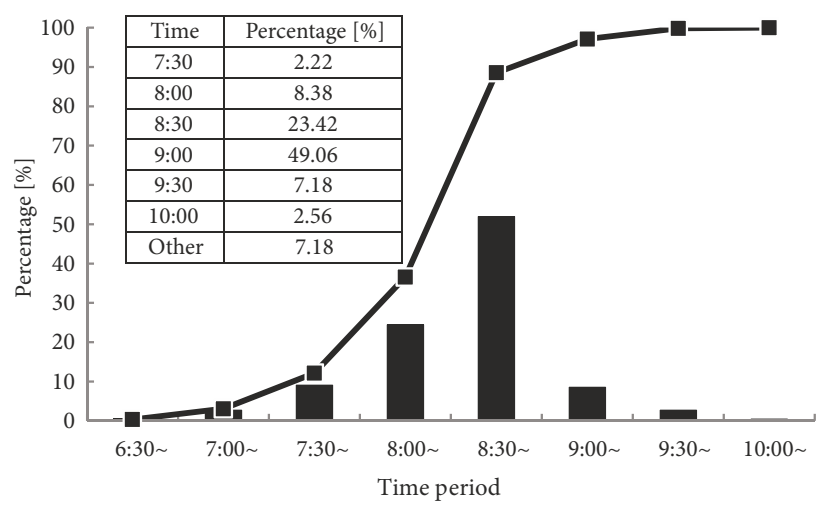

Figure 4. The distribution of WST

Table 1. Commuters' socioeconomic and travel characteristics

\begin{tabular}{|c|c|c|c|}
\hline Items & Classification & Frequency & Percentage $[\%]$ \\
\hline \multirow{2}{*}{ Gender } & male & 266 & 45.47 \\
\hline & female & 319 & 54.53 \\
\hline \multirow{4}{*}{ Age } & younger than 18 years old & 10 & 1.71 \\
\hline & $18 \ldots 40$ years old & 513 & 87.69 \\
\hline & $41 \ldots 60$ years old & 61 & 10.43 \\
\hline & older than 61 years old & 1 & 0.17 \\
\hline \multirow{2}{*}{ The frequency of travelling by metro in one week } & $1 \ldots 2$ times & 61 & 10.43 \\
\hline & more than 3 times & 524 & 89.57 \\
\hline \multirow{2}{*}{ The time period of travelling by metro in one week } & only weekdays & 163 & 27.86 \\
\hline & weekdays and weekends & 422 & 72.14 \\
\hline \multirow{2}{*}{ The purpose of the first metro trip on the latest weekday } & go to work & 568 & 97.09 \\
\hline & go to school & 17 & 2.91 \\
\hline
\end{tabular}


ignored in the valuation of arrival time. The average of SCDET is between WST and ALAT, which is $3.99 \mathrm{~min}$ earlier than the average of ALAT and 3.27 min later than the one of WST. $79.83 \%$ of respondents start to consider departing earlier when time is earlier than ALAT, and the ALAT of $59.32 \%$ of respondents is earlier than their WST. Compared with the situation on the early-side, the situation on the late-side seems to imply that commuters tend to be more cautious when they are likely to be late.

The average of PAT is 10.12 min earlier than the average of WST. The lower standard deviation of the difference between WST and PAT indicates that the relationship between these two alternative points is more stable. $98.80 \%$ of respondents think PAT is earlier than WST, and the other $1.20 \%$ of respondents think PAT equals to WST. This evidence demonstrates that commuters tend to arrive at work place/school in advance for preparation or other reasons.

Table 4 shows that the ATT of $64.27 \%$ of respondents is not later than PAT, which including $18.97 \%$ of respondents whose AAT is just their PAT. The remaining 35.73\% of respondents arrive at work place or school later than PAT. Most of respondents $(79.29 . .93 .16 \%)$ have a gain because their AAT is between EAT and LAT, no matter which alternative is selected as a reference point. Moreover, more commuters have an early-side gain. The difference between AAT and PAT is no more than $10 \mathrm{~min}$ for $85.30 \%$ of commuters.

Because the majority of respondents experience a gain, so they are less likely to switch their departure times. 497 respondents intend to keep the same departure time in the next weekday. Only 88 respondents choose to switch their departure time, accounting for $15.04 \%$.

\section{Estimation and discussion}

\subsection{Estimation method}

The parameters are estimated by applying binary probit model (Ben-Akiva, Lerman 1985). The probability of switching departure time is expressed as a function of arrival time value. A commuter will maintain his/her departure time when the utility is greater than zero, and the commuter will switch the departure time when the utility is negative. So the probability of these choices can be written in Equations (4) and (5):

Table 2. The alternative reference points of arrival time valuation

\begin{tabular}{|l|c|c|c|c|}
\hline \multicolumn{1}{|c|}{ Reference point } & Mean & Min & Max & Standard deviation \\
\hline AEAT, $t_{e 1}$ & $8: 24$ & $6: 20$ & $9: 55$ & 33.43 \\
\hline SCDLT, $t_{e 2}$ & $8: 18$ & $6: 30$ & $9: 50$ & 33.03 \\
\hline PAT, $t_{p}$ & $8: 38$ & $6: 35$ & $10: 00$ & 30.80 \\
\hline WST, $t_{w}$ & $8: 48$ & $6: 45$ & $10: 30$ & 29.85 \\
\hline ALAT, $t_{l 1}$ & $8: 55$ & $6: 45$ & $11: 00$ & 32.83 \\
\hline SCDET, $t_{l 2}$ & $8: 51$ & $6: 45$ & $10: 40$ & 32.76 \\
\hline
\end{tabular}

Table 3. The difference between alternative reference points of arrival time valuation

\begin{tabular}{|l|c|c|c|c|c|c|}
\hline \multirow{2}{*}{\multicolumn{1}{c|}{ Difference }} & \multicolumn{2}{c|}{ ANOVA } & \multirow{2}{*}{ Mean } & \multirow{2}{*}{ Min } & \multirow{2}{*}{ Max } & \multirow{2}{*}{ Standard deviation } \\
\cline { 2 - 6 } & $\mathrm{F}$ & Sigma & & & & \\
\hline SCDLT - AEAT, $t_{e 2}-t_{e 1}$ & 8.784 & $0.003^{* *}$ & -5.76 & -60.00 & 70.00 & 12.05 \\
\hline WST - PAT, $t_{w}-t_{p}$ & 32.555 & $0.000^{* *}$ & 10.12 & 0.00 & 50.00 & 5.99 \\
\hline ALAT - WST, $t_{l 1}-t_{w}$ & 15.691 & $0.000^{* *}$ & 7.27 & 0.00 & 90.00 & 10.23 \\
\hline SCDET - WST, $t_{l 2}-t_{w}$ & 3.189 & $0.074^{*}$ & 3.27 & -20.00 & 60.00 & 11.12 \\
\hline SCDET - ALAT, $t_{l 2}-t_{l 1}$ & 4.314 & $0.037^{* *}$ & -3.99 & -70.00 & 45.00 & 11.06 \\
\hline
\end{tabular}

Notes: ${ }^{*}$ difference is significant at $10 \%$ level; ${ }^{* *}$ difference is significant at $5 \%$ level.

Table 4. The distribution of commuters' AAT

\begin{tabular}{|c|c|c|c|c|c|}
\hline \multirow{3}{*}{$\begin{array}{l}\text { Early-side } \\
\text { arrivals, } \\
t_{a} \leq t_{p}\end{array}$} & \multirow{3}{*}{$376(64.27 \%)$} & Reference point & Alternatives & $t_{d}<t_{a} \leq t_{E}$ & $t_{E}<t_{a} \leq t_{p}$ \\
\hline & & \multirow{2}{*}{$\mathrm{EAT}, t_{E}$} & AEAT, $t_{e 1}$ & 89 (15.21\%) & $287(49.06 \%)$ \\
\hline & & & SCDLT, $t_{e 2}$ & $28(4.79 \%)$ & $348(59.49 \%)$ \\
\hline \multirow{4}{*}{$\begin{array}{l}\text { Late-side } \\
\text { arrivals, } \\
t_{a}>t_{p}\end{array}$} & \multirow{4}{*}{209 (35.73\%) } & Reference point & Alternatives & $t_{p}<t_{a} \leq t_{L}$ & $t_{a}>t_{L}$ \\
\hline & & \multirow{3}{*}{$\mathrm{LAT}, t_{L}$} & WST, $t_{w}$ & $171(29.23 \%)$ & $38(6.50 \%)$ \\
\hline & & & ALAT, $t_{l 1}$ & $197(33.68 \%)$ & $12(2.05 \%)$ \\
\hline & & & $\mathrm{SCDET}, t_{l 2}$ & $192(32.82 \%)$ & $17(2.91 \%)$ \\
\hline
\end{tabular}




$$
\begin{aligned}
& p(N S)=p(d=1)=p\left(U\left(V\left(t_{a}\right)\right)>0\right) ; \\
& p(S)=p(d=0)=p\left(U\left(V\left(t_{a}\right)\right)<0\right),
\end{aligned}
$$

where: $p(N S)$ is the probability of not switching departure time; $p(S)$ is the probability of switching departure time; $U\left(V\left(t_{a}\right)\right)$ is the utility function of binary probit model; $d=1$ if the commuter maintain his/her departure time and $d=0$, otherwise.

Utility function is a function of arrival time value, which can be divided into two types. It is assumed that commuters have no reluctance to original departure time in the first type, then $U\left(V\left(t_{a}\right)\right)=V\left(t_{a}\right)$, as shown in Equations (1)-(3). In the second type, reluctance factors are introduced. When the reference point of commuters is OAT, then the reluctance is a part of the value of OAT $\left(\gamma_{1}\right.$ and $\left.\gamma_{2}\right)$, so the utility function of F2 has no change. A reluctance factor is only added into utility functions when the reference point is EAT or LAT, after that the utility functions of F1 and F3 change into Equations (6) and (7):

$$
\begin{gathered}
U\left(V_{1}\left(t_{a}\right)\right)= \begin{cases}c_{1}+\beta_{1} \cdot\left(t_{E}-t_{a}\right)^{\alpha_{1}}+\varepsilon_{e}^{L}, & t_{d}<t_{a} \leq t_{E} ; \\
c_{1}+\beta_{2} \cdot\left(t_{a}-t_{E}\right)^{\alpha_{2}}+\varepsilon_{e}^{G}, & t_{E}<t_{a} \leq t_{O} ; \\
c_{2}+\beta_{3} \cdot\left(t_{L}-t_{a}\right)^{\alpha_{3}}+\varepsilon_{l}^{G}, & t_{O}<t_{a} \leq t_{L} ; \\
c_{2}+\beta_{4} \cdot\left(t_{a}-t_{L}\right)^{\alpha_{4}}+\varepsilon_{l}^{L}, & t_{a} \geq t_{L} ;\end{cases} \\
U\left(V_{3}\left(t_{a}\right)\right)= \begin{cases}\gamma_{2}+\beta_{7} \cdot\left(t_{O}-t_{a}\right)^{\alpha_{7}}+\varepsilon_{e}, & t_{d}<t_{a} \leq t_{O} ; \\
c_{2}+\beta_{8} \cdot\left(t_{L}-t_{a}\right)^{\alpha_{8}}+\varepsilon_{l}^{G}, & t_{O}<t_{a}<t_{L} ; \\
c_{2}+\beta_{9} \cdot\left(t_{a}-t_{L}\right)^{\alpha_{9}}+\varepsilon_{l}^{L}, & t_{a} \geq t_{L},\end{cases}
\end{gathered}
$$

where: $c_{1}$ is a reluctance factor for early-side arrivals; $c_{2}$ is a reluctance factors for late-side arrivals; both reluctance factors should be positive.

Since utility functions are non-linear segmented functions, if a logarithmic transformation is adopted, the assumption of normally-distributed error terms would be broken. So a method combining maximum-likelihood with limited enumeration is proposed. The exponents of arrival time value function are set to be a group of fixed values within a given range. This step transforms nonlinear functions into linear functions, so the maximum likelihood method becomes practicable for estimation. Based on previous studies, the value range of each exponent was set to be $(0,2]$, with step size 0.05 . So for each model within a valuation framework, the size of estimation result depends on the number of parameter $\alpha$ in its value function. If the number of $\alpha$ is $n$, then there are $40^{n}$ sets of estimated parameters. Because early-side arrivals and late-side arrivals do not affect each other in F1 and F3, so these two parts of utility functions can be estimated separately to improve efficiency. The entire framework of estimation is illustrated in Figure 5. There are 12 models within F1, 2 models within F2 and 6 models within F3 to be estimated.

Two kinds of significance tests were performed on each estimation result. The first kind is economic significance test, which examines whether the signs of parameters are consistent with the proposed theory. Furthermore, EAT and LAT are calculated by back-stepping in F2 and F3. It is also tested whether the difference between them and PAT is within a reasonable range. The upper limit of the range is defined as the 90 percentile value of the difference according to the collected data, which is $35 \mathrm{~min}$ for EAT and $30 \mathrm{~min}$ for LAT. The second kind is statistical significance test, which mainly examines whether all parameters pass $t$-test at the $5 \%$ level. Within each valuation framework, only the estimation results passing two tests are picked out to be compared with each other. Then the one with maximal log-likelihood was chosen as the best estimation result of the model within this valuation framework.

\subsection{Estimation result}

Parameters $\beta_{i}(i=1, \ldots, 9)$ are weights, which represent the importance of value in different segments to commuter, while $c_{i}(i=1,2)$ are the factors to reflect commuters' reluctance to their original departure time. Only when $\beta$ passes significance tests and the sign is in accordance with

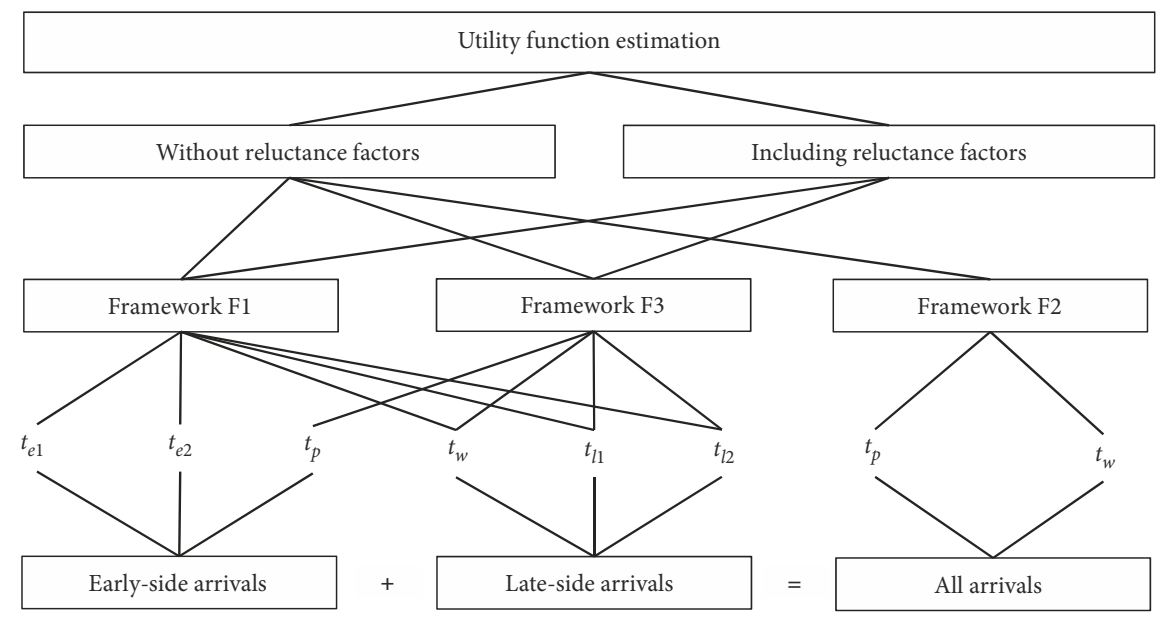

Figure 5. A framework of utility function estimation 
the inference of valuation framework, the corresponding alternative is regarded as a reasonable reference point within this framework. Moreover, the pass of $c$ implies that commuters really add extra value on original choice.

If early-side arrivals take EAT as reference point, none of estimation results pass two significance tests at the same time, regardless of which alternative is chosen. Figure 6 shows that without a reluctance factor, most of $\beta_{1}$ and $\beta_{2}$ pass statistical significance test, however both of them are positive. Based on the framework F1, the arrival time value decreases with the increase of its deviation from EAT. So $\beta_{1}$ should be negative, but it is violated by the estimation result. When reluctance factor $c_{1}$ is considered, the signs of $\beta_{1}$ and $\beta_{2}$ turn to right, but two parameters cannot pass $t$-test simultaneously. If the reference point of earlyside arrivals changes to PAT, then parameters $\gamma_{2}$ and $\beta_{7}$ in most of estimation results are significant, economically and statistically.

Figure 7 presents that one or both of $\beta_{3}$ (or $\beta_{8}$ ) and $\beta_{4}$ (or $\beta_{9}$ ) fail $t$-test if late-side arrivals take WST or ALAT as their reference point, no matter a reluctance factor is included or not and how much exponents are. If the reference point of late-side arrivals changes to SCDET, both $\beta_{3}$ (or $\beta_{8}$ ) and $\beta_{4}$ (or $\beta_{9}$ ) in some estimation results are significant. Meanwhile, reluctance factor $c_{2}$ in these results also pass $t$-test and have positive values, which is in line with the assumption.

For all arrivals, if WST is the only reference point, none of estimation results pass statistical significance test because the t statistics of $\beta_{5}$ are less than 1.96. When the reference point is PAT, then some estimation results pass both significance tests (Figure 8).

Thus, by combining the situation of early-side arrivals and late-side arrivals, we can find that all the models within F1 fail significance tests. Within F2, the models with WST as OAT are unable to pass significant tests. The best estimation comes out when PAT is the only reference point. Within F3, a model with PAT and SCDET as reference points has the best estimation result. Table 5 reports both of best estimation results. The best result of $\mathrm{F} 2$ shows the value of PAT is 2.255. $\beta_{6}$ is greater than $\beta_{5}$ in absolute values, while $\alpha_{5}$ and $\alpha_{6}$ are between 0 and 1 . According to the best result of F3, the value of PAT is 2.153 , which is close to the one in F2. For late-side arrivals, reluctance factor $c_{3}$ equals to 0.383 , and it is significant at $1 \%$ level. The absolute values of $\beta_{9}$ and $\alpha_{8}$ are larger than $\beta_{8}$ and $\alpha_{8}$, respectively. The performance of best functions within two valuation frameworks presents that the one within F2 fits the data a little better than the one within F3, with a hit rate $85.64 \%$. a)

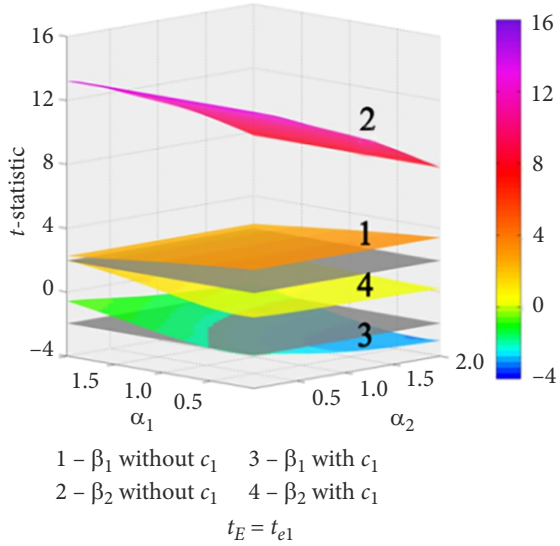

b)

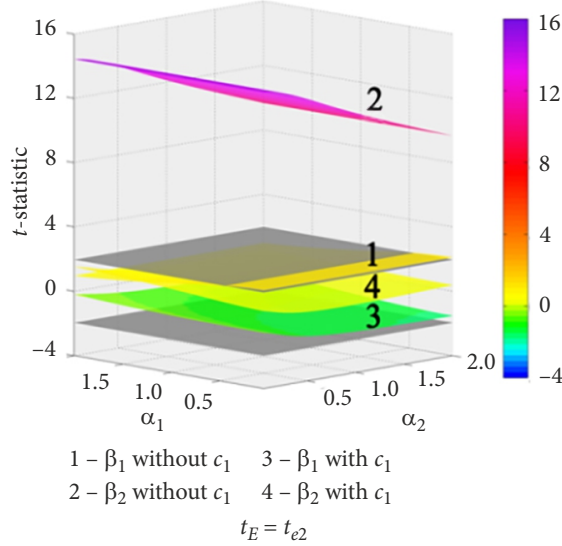

c)

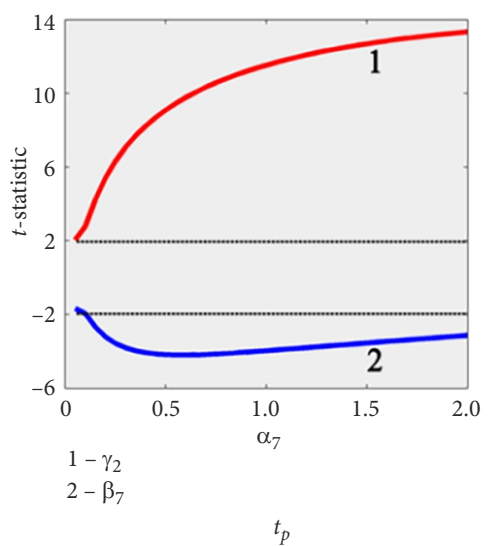

Figure 6. Statistical significance test result of estimation result (early-side arrivals)
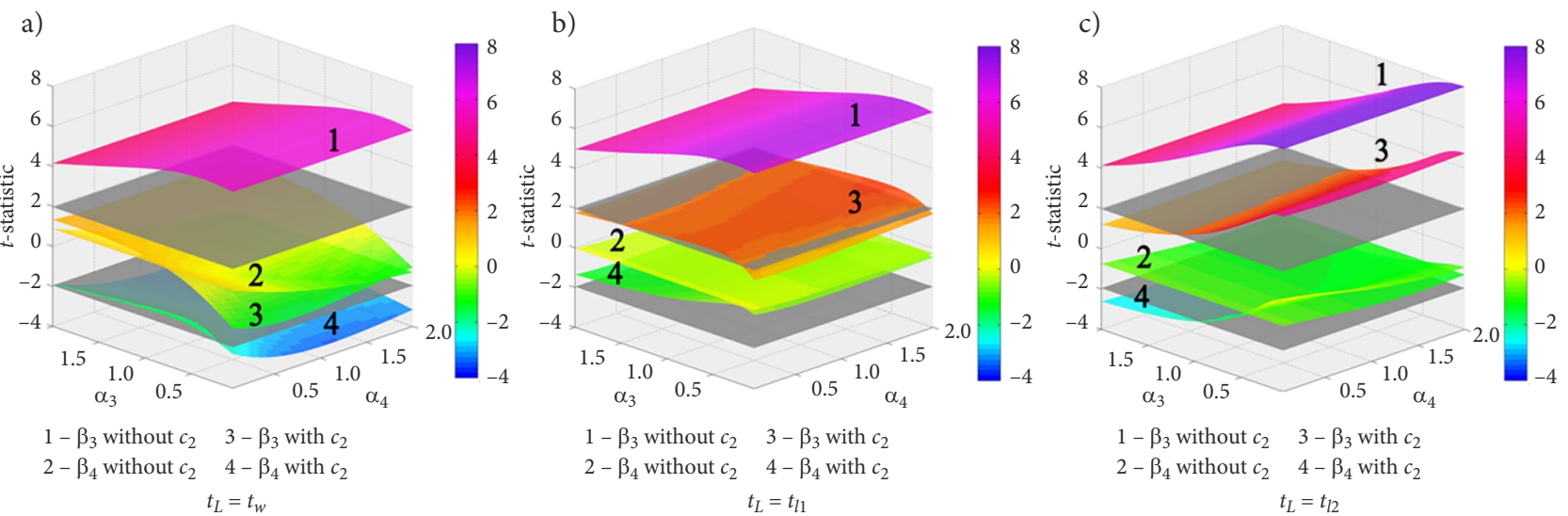

Figure 7. Statistical significance test result of estimation result (late-side arrivals) 

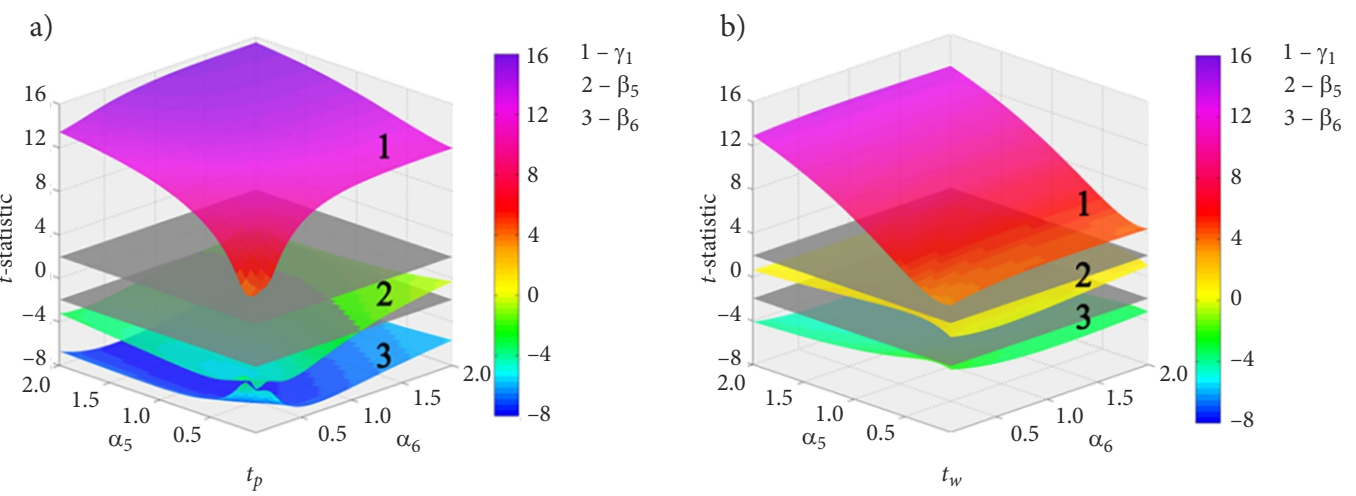

Figure 8. Statistical significance test result of estimation result (all arrivals)

Table 5. Best estimation results within each valuation framework

\begin{tabular}{|c|c|c|c|c|c|c|c|c|c|c|c|}
\hline & \multicolumn{3}{|c|}{ Reference points } & \multirow{2}{*}{ Region } & \multirow{2}{*}{ Parameter } & \multirow{2}{*}{ Variable } & \multirow{2}{*}{ Value } & \multirow{2}{*}{$t$-stat } & \multirow{2}{*}{$\log L$} & \multirow{2}{*}{ Pseudo $R^{2}$} & \multirow{2}{*}{ Hit rate $[\%]$} \\
\hline & EAT, $t_{E}$ & OAT, $t_{O}$ & $\mathrm{LAT}, t_{L}$ & & & & & & & & \\
\hline F1 & \multicolumn{11}{|c|}{ None of estimation results pass both economic and statistical significance tests } \\
\hline \multirow{5}{*}{ F2 } & \multirow{5}{*}{-} & \multirow{5}{*}{ PAT, $t_{p}$} & \multirow{5}{*}{-} & \multirow{5}{*}{ All arrivals } & $\gamma_{1}$ & - & 2.255 & 10.349 & \multirow{5}{*}{-210.103} & \multirow{5}{*}{0.4819} & \multirow{5}{*}{85.64} \\
\hline & & & & & $\beta_{5}$ & $t_{O}-t_{a}$ & -0.384 & -4.747 & & & \\
\hline & & & & & $\beta_{6}$ & $t_{a}-t_{O}$ & -0.956 & -7.349 & & & \\
\hline & & & & & $\alpha_{5}$ & $t_{O}-t_{a}$ & 0.50 & - & & & \\
\hline & & & & & $\alpha_{6}$ & $t_{a}-t_{O}$ & 0.30 & - & & & \\
\hline \multirow{8}{*}{ F3 } & \multirow{8}{*}{-} & \multirow{8}{*}{$\mathrm{PAT}, t_{p}$} & \multirow{8}{*}{ SCDET, $t_{l 2}$} & \multirow{3}{*}{$\begin{array}{l}\text { Early-side } \\
\text { arrivals }\end{array}$} & $\gamma_{2}$ & - & 2.153 & 9.464 & \multirow{8}{*}{-210.458} & \multirow{8}{*}{0.4809} & \multirow{8}{*}{85.13} \\
\hline & & & & & $\beta_{7}$ & $t_{a}-t_{O}$ & -0.311 & -4.192 & & & \\
\hline & & & & & $\alpha_{7}$ & $t_{a}-t_{O}$ & 0.55 & - & & & \\
\hline & & & & \multirow{5}{*}{$\begin{array}{l}\text { Late-side } \\
\text { arrivals }\end{array}$} & $c_{3}$ & - & 0.383 & 2.634 & & & \\
\hline & & & & & $\beta_{8}$ & $t_{L}-t_{a}$ & 0.044 & 2.658 & & & \\
\hline & & & & & $\beta_{9}$ & $t_{a}-t_{L}$ & -0.087 & -1.962 & & & \\
\hline & & & & & $\alpha_{8}$ & $t_{L}-t_{a}$ & 0.85 & - & & & \\
\hline & & & & & $\alpha_{9}$ & $t_{a}-t_{L}$ & 1.20 & - & & & \\
\hline
\end{tabular}

\subsection{Discussion}

The failure of all the models within F1 to pass economic and statistical significance tests indicates that it is not suitable for the arrival time valuation of commuters in urban rail transit. The combination of EAT and LAT does not appear to be the best reference points. Within the framework F2, the models with WST as the only reference point also cannot pass significance tests. Although schedule delay is used as a representative of arrival time value in many literatures, the result shows that it does not properly reflect the arrival time valuation of urban rail transit commuters. Instead, the value functions within F2 and F3 have preferable estimation results.

The model with PAT as OAT shows best within F2. The weight representing the importance of value on late-side $\beta_{6}$ has a higher absolute value than the one on early-side $\beta_{5}$, which means commuters respond asymmetrically to arrivals on different sides and they attach more disutility to being late. The estimation of $\alpha_{5}$ and $\alpha_{6}$ demonstrates that the value function is non-linear. The curves in quasi- gain regions are convex, which is consistent with prospect theory. Because the reference point of these regions is PAT. Commuters evaluate arrival time with respect to the decline from the gain at PAT. Although their arrival time value may be positive, they feel perceived loss and exhibit risk seeking. It can be reckoned that when a commuter arrives $34.48 \mathrm{~min}$ earlier or $17.46 \mathrm{~min}$ later than PAT, arrival time value reduces to zero. The difference between calculated EAT and PAT is almost twice as much as the one between calculated PAT and LAT, which supports the inference that commuters tend to be more cautious when they are likely to be late.

The best estimation result within $\mathrm{F} 3$ is the model with PAT as EAT and SCDET as PAT. The reluctance to original departure time is proved to be acceptable, which means when a commuter arrives at his/her perceived LAT, the commuter would not switch the departure time next time. Based on the value of $c_{3}$, the actual indifference point is 3.43 min later than SCDET. Asymmetrical respond to arrivals on different sides is verified again by this model. The calculated EAT is 33.70 min earlier than PAT, and the 
average difference between actual indifference point and PAT is $16.82 \mathrm{~min}$, nearly half of the difference between EAT and PAT. According to the estimation of $\alpha_{8}$ and $\alpha_{9}$, when the reference point is LAT, the curves are concave for both gains and losses, which means commuters would like to averse risks. It seems that for a commuter who is late for work/school, marginal penalty increases with the deviation from WST. Last but not least, the relationship between $\beta_{8}$ and $\beta_{9}$ as well as the one between $\alpha_{8}$ and $\alpha_{9}$ indicate that commuters respond asymmetrically not only to arrivals on different sides, but also to arrivals on the same side. For late-side arrivals, the value function is steeper when arrival time is later than SCDET.

The comparison between the best estimation results suggests that the framework with PAT as the only reference point better depicts the arrival time valuation of urban rail transit commuters. According to this valuation framework, a commuter obtains his/her maximum value when arrival time is PAT; otherwise, arrival time value decreases with the difference between AAT and PAT. Although the concavity of loss region within F3 violates the property of prospect theory, all signs of parameters and the convexity (or concavity) of value functions in quasigain and gain regions suggest that the arrival time value function of commuter rail transit users is basically in accordance with prospect theory.

\section{Conclusions}

The arrival time value is one of the most important influence factors of the departure time choice of urban rail commuters. Different from most of previous researches, this paper does not use schedule delay to represent arrival time value of commuters. Instead, it introduces the reference point approach in prospect theory to study the valuation of commuter arrival time. Three frameworks with various reference points are proposed based on different assumptions of commuter behaviour. Each kind of reference point (EAT, PAT and OAT) has several alternatives. All the combinations of valuation framework and reference point alternatives are tested and compared with the data of commuters in Shanghai Metro System. The estimation method combines maximum-likelihood with limited enumeration. Results provide a clear answer to the questions, which are asked at the beginning of the paper.

First, the schedule delay used by most of studies cannot represent arrival time value of urban rail transit commuters. Instead, commuters value their arrival time according to its deviation from PAT. The collected data of Shanghai commuters shows that average PAT is about 10 min earlier than WST. Second, the estimated values of exponents indicate that commuters judge their arrival time in a non-linear way. When PAT is the only reference point, the curves are convex in quasi-gain regions, which means urban rail commuters become risk seekers when they experience loss. At last, the higher weight on late-side suggests that commuters attach more disutility to being late, so they are more cautious when they tend to be late.
Basically, the reference point hypothesis of prospect theory is applicable to arrival time valuation of urban rail commuters. The analysis and quantification of arrival time value presented in this paper can be a basis for establishing departure time choice model for commuters in urban rail transit. The result will provide support for the decision-making of policies to relieve overcrowding and improve the quality of urban rail transit. The trade-off among arrival time value, the additional psychological pressure caused by in-vehicle crowding, and time uncertainty will be studied in the future.

\section{Funding}

This research received no specific grant from any funding agency in the public, commercial, or not-for-profit sectors.

\section{Contribution}

Xiafei Ye and Yan Cheng contributed to the conception of the study. Yan Cheng contributed significantly to the acquisition and analysis of data, and wrote the manuscript. Zhi Wang helped perform the analysis with constructive discussions. Xiafei Ye revised the manuscript critically for important intellectual content.

\section{Disclosure statement}

All the authors have no competing financial, professional, or personal interests from other parties.

\section{References}

Abkowitz, M. D. 1980. The Impact of Service Reliability on Work Travel Behavior. Doctoral Dissertation. Massachusetts Institute of Technology, United States, 265 p. Available from Internet: https://dspace.mit.edu/bitstream/handle/1721.1/ 16079/07640839-MIT.pdf

Arnott, R.; De Palma, A.; Lindsey, R. 1990. Departure time and route choice for the morning commute, Transportation Research Part B: Methodological 24(3): 209-228.

https://doi.org/10.1016/0191-2615(90)90018-T

Ben-Akiva, M. E.; Lerman, S. R. 1985. Discrete Choice Analysis: Theory and Application to Travel Demand. MIT Press. 412 p.

Chang, G.-L.; Mahmassani, H. S. 1989. The dynamics of commuting decision behaviour in urban transportation networks, in Travel Behaviour Research: 5th International Conference on Travel Behaviour, October 1987, Aix-en-Provence, France, 15-26.

De Palma, A.; Ben-Akiva, M.; Lefevre, C.; Litinas, N. 1983. Stochastic equilibrium model of peak period traffic congestion, Transportation Science 17(4): 430-453. https://doi.org/10.1287/trsc.17.4.430

De Palma, A.; Kilani, M.; Proost, S. 2015. Discomfort in mass transit and its implication for scheduling and pricing, Transportation Research Part B: Methodological 71: 1-18. https://doi.org/10.1016/j.trb.2014.10.001

De Palma, A.; Lindsey, R.; Monchambert, G. 2017. The economics of crowding in rail transit, Journal of Urban Economics 101: 106-122. https://doi.org/10.1016/j.jue.2017.06.003 
Feng, J.; Mao, B.; Chen, Z.; Bai, Y.; Li, M. 2013. A Departure time choice for morning commute considering train capacity of a rail transit line, Advances in Mechanical Engineering 5: 582703. https://doi.org/10.1155/2013/582703

Han, L.; Ukkusuri, S.; Doan, K. 2011. Complementarity formulations for the cell transmission model based dynamic user equilibrium with departure time choice, elastic demand and user heterogeneity, Transportation Research Part B: Methodological 45(10): 1749-1767.

https://doi.org/10.1016/j.trb.2011.07.007

Harada, C.; Iwakura, S.; Morichi, S. 2002. Analysis and modeling of commuters' departure time in urban railway network, Proceedings of Infrastructure Planning 26: 1-4. (in Japanese).

Hendrickson, C.; Plank, E. 1984. The flexibility of departure times for work trips, Transportation Research Part A: General 18(1): 25-36. https://doi.org/10.1016/0191-2607(84)90091-8

Huang, H.-J.; Lam, W. H. K. 2002. Modeling and solving the dynamic user equilibrium route and departure time choice problem in network with queues, Transportation Research Part B: Methodological 36(3): 253-273.

https://doi.org/10.1016/S0191-2615(00)00049-7

Huang, Y. 2010. Research on Urban Rail Transit Passenger Flow Assignment Model and Algorithm. MSc Thesis. Beijing Jiaotong University, China, $53 \mathrm{p}$. (in Chinese).

Ieda, H.; Tsuchiya, K.; Phan, L. B.; Okamura, T. 2002. Development of the commuter demand concentration model based on a time-space network scheme, Journal of Japan Society of Civil Engineers (702): 65-79.

https://doi.org/10.2208/jscej.2002.702_65 (in Japanese).

Iwakura, S.; Harada, C. 2005. A model system of departure time choice for commuter trips by metropolitan railway, Transport Policy Studies' Review 8: 4-15.

Iwakura, S.; Harada, C.; Suzuki, S. 2003. Comparative analysis of choice set for commuting time of day choice model in urban railway networks, Infrastructure Planning Review 20(3): 485-492. https://doi.org/10.2208/journalip.20.485

Jou, R.-C.; Kitamura, R. 2002. Commuter departure time choice: a reference-point approach, in 9th Meeting of the Euro Working Group on Transportation, 10-13 June 2002, Bari, Italy, 149-155. Available from Internet: http://www.iasi.cnr.it/ewgt/13conference/26_jou.pdf

Jou, R.-C.; Kitamura, R.; Weng, M.-C.; Chen, C.-C. 2008. Dynamic commuter departure time choice under uncertainty, Transportation Research Part A: Policy and Practice 42(5): 774-783. https://doi.org/10.1016/j.tra.2008.01.017

Kahneman, D.; Tversky, A. 1979. Prospect theory: an analysis of decision under risk, Econometrica 47(2): 263-291. https://doi.org/10.2307/1914185

Kristoffersson, I. 2007. Implementation of model for departure time choice, in TRISTAN VI - Sixth Triennial Symposium on Transportation Analysis, 10-15 June 2007, Phuket Island, Thailand, 1-19.

Liu, J. 2012. Transfer-based Urban Rail Transit Flow Distribution Modeling and Empirical Study. Doctoral Dissertation. Beijing Jiaotong University, China, 119 p. (in Chinese).

Liu, X. 2013. Research on the Dynamic Flow Assignment Model Based on Train Schedule for Urban Subway Network. Doctoral Dissertation. Changan University, China, 135 p. (in Chinese).

Mahmassani, H. S.; Chang, G.-L. 1986. Experiments with departure time choice dynamics of urban commuters, Transportation Research Part B: Methodological 20(4): 297-320. https://doi.org/10.1016/0191-2615(86)90045-7

Noland, R. B.; Small, K. A. 1995. Travel-time uncertainty, departure time choice, and the cost of morning commutes, Transportation Research Record 1493: 150-158.
Peer, S.; Knockaert, J.; Verhoef, E. T. 2016. Train commuters' scheduling preferences: Evidence from a large-scale peak avoidance experiment, Transportation Research Part B: Methodological 83: 314-333.

https://doi.org/10.1016/j.trb.2015.11.017

Poon, M. H.; Wong, S. C.; Tong, C. O. 2004. A dynamic schedule-based model for congested transit networks, Transportation Research Part B: Methodological 38(4): 343-368. https://doi.org/10.1016/S0191-2615(03)00026-2

Ran, B.; Hall, R. W.; Boyce, D. E. 1996. A link-based variational inequality model for dynamic departure time/route choice, Transportation Research Part B: Methodological 30(1): 31-46. https://doi.org/10.1016/0191-2615(95)00010-0

Senbil, M.; Kitamura, R. 2004. Reference points in commuter departure time choice: a prospect theoretic test of alternative decision frames, Journal of Intelligent Transportation Systems: Technology, Planning, and Operations 8(1): 19-31. https://doi.org/10.1080/15472450490437726

Simon, H. A. 1955. A behavioral model of rational choice, The Quarterly Journal of Economics 69(1): 99-118. https://doi.org/10.2307/1884852

Small, K. A. 1982. The scheduling of consumer activities: work trips, The American Economic Review 72(3): 467-479.

Soyama, Y.; Kaneko, Y.; Kato, H. 2010. Departure time choice under the condition of daily service delay in urban railway, Proceedings of Infrastructure Planning 41: 1-4. (in Japanese).

Tian, Q.; Huang, H. 2004. An equilibrium ride model for subway passengers with arrival early penalty, Journal of Transportation Systems Engineering and Information Technology 4(4): 108-112. (in Chinese).

Tian, Q.; Huang, H.-J.; Yang, H. 2007. Equilibrium properties of the morning peak-period commuting in a many-to-one mass transit system, Transportation Research Part B: Methodological 41(6): 616-631. https://doi.org/10.1016/j.trb.2006.10.003

Van de Kaa, E. J. 2010. Applicability of an extended prospect theory to travel behaviour research: a meta-analysis, Transport Reviews 30(6): 771-804.

https://doi.org/10.1080/01441647.2010.486907

Vickrey, W. S. 1969. Congestion theory and transport investment, The American Economic Review 59(2): 251-260.

Wu, W.; Huang, H. 2009. Model of subway commuters' departure time choice with in-carriage congestion and arrival early/late penalty, Journal of Transportation Systems Engineering and Information Technology 9(1): 128-132. (in Chinese).

Wu, X.-Y.; Liu, C.-Q. 2004. Traffic equilibrium assignment model specially for urban railway network, Journal of Tongji University (Natural Science) 32(9): 1158-1162. (in Chinese).

Yang, D. 2013. Research on Schedule-based Rail Transit Passenger Flow Assignment. MSc Thesis. Beijing Jiaotong University, China, 72 p. (in Chinese).

Yang, G.; Liu, X. 2018. A commuter departure-time model based on cumulative prospect theory, Mathematical Methods of Operations Research 87(2): 285-307.

https://doi.org/10.1007/s00186-017-0619-8

Yang, H.; Meng, Q. 1998. Departure time, route choice and congestion toll in a queuing network with elastic demand, Transportation Research Part B: Methodological 32(4): 247-260. https://doi.org/10.1016/S0191-2615(97)00041-6

Yang, H.; Tang, Y. 2018. Managing rail transit peak-hour congestion with a fare-reward scheme, Transportation Research Part B: Methodological 110: 122-136.

https://doi.org/10.1016/j.trb.2018.02.005 